\title{
An elevated blood glucose level and increased incidence of gestational diabetes mellitus in pregnant women with latent toxoplasmosis
}

\author{
Š́́rka Kan̆ková $^{1}$, Jaroslav Flegr ${ }^{1}$ and Pavel Calda ${ }^{2}$ \\ ${ }^{1}$ Department of Philosophy and History of Science, Faculty of Science, Charles University, Prague, Czech Republic; \\ ${ }^{2}$ Department of Obstetrics and Gynaecology, General University Hospital and First Faculty of Medicine, Charles University, Prague, \\ Czech Republic
}

\begin{abstract}
About $30-50 \%$ of the world human population are infected with the protozoan parasite Toxoplasma gondii (Nicolle et Manceaux, 1908). Latent toxoplasmosis has many specific behavioural and physiological effects on the human body and influences the course of pregnancy, including secondary sex ratio of children of infected mothers. It was suggested that an increased concentration of glucose could be the proximate cause of increased sex ratio. There are some indirect indications of possible association between toxoplasmosis and certain forms of diabetes. Here we searched for a possible link between latent toxoplasmosis and the level of glucose in the blood. In a cross-sectional study, we found that pregnant women with latent toxoplasmosis had significantly higher blood glucose levels during the oral glucose tolerance test $(\mathrm{n}=191, \mathrm{p}=0.010$; the level of fasting plasma glucose: mean $=5.04 \mathrm{mmol} / 1 \mathrm{vs}$ mean $=4.88 \mathrm{mmol} / \mathrm{l}$; blood glucose level at 1 hour mean $=7.73 \mathrm{mmol} / 1 \mathrm{vs}$ mean $=6.89 \mathrm{mmol} / \mathrm{l}$ and blood glucose level at two hours mean $=6.43 \mathrm{mmol} / \mathrm{l} v \mathrm{~s}$ mean $=5.74 \mathrm{mmol} / \mathrm{l})$ and higher prevalence $(19.5 \%)$ of gestational diabetes mellitus $(\mathrm{n}=532, \mathrm{p}=0.033$, odds ratio $=1.78)$ in the $24-28$ th gestational weeks than $T$. gondii-free women $(12.0 \%)$. Increased level of glucose and increased incidence of gestational diabetes mellitus could have considerable clinical impact as contributors to the development of the metabolic syndrome and type 2 diabetes in T. gondii-infected women. Our results also brought the first empirical support for the hypothesis that the glucose concentration may play a role in $T$. gondii-associated offspring sex ratio shifts.
\end{abstract}

Keywords: parasite, GDM, oral glucose tolerance test, sex ratio, risk factors, Toxoplasma gondii

Toxoplasmosis, an endemic zoonosis caused by a coccidian protozoan, Toxoplasma gondii (Nicolle et Manceaux, 1908), is probably the most widespread human parasite in developed countries. The prevalence of latent toxoplasmosis in the population ranges from $20 \%$ to $80 \%$ depending on various environmental and sociological factors, including the number of cats in the environment, latitude, moisture, hygienic standards and kitchen habits (Tenter et al. 2000). In immunocompetent humans, postnatally acquired toxoplasmosis is either inapparent, without any clinical signs, or accompanied by cervical lymphadenopathy with fever, joint pain, headache and tiredness (Beattie 1982, Jones et al. 2001). Acute disease promoted by rapidly dividing tachyzoites in cells of many tissues often progresses spontaneously to the latent phase characterised by probably permanent presence of slowly dividing bradyzoites in tissue cysts in various organs of the host.

Latent toxoplasmosis is usually considered to pose no health threat to immunocompetent individuals; however, it is accompanied by specific changes in the psychomotor performance, behaviour and personality profile (Flegr et al. 1996, Lindová et al. 2006, Flegr 2013). A recent correlation study performed on a set of 88 countries has also shown that the prevalence of toxoplasmosis explains about $13 \%$ of the variation in the rates of congenital abnormalities between the countries (Flegr et al. 2014).

Unlike acute toxoplasmosis in pregnancy, which has been described to damage significantly the foetal development (Wolf et al. 1939, Tenter et al. 2000), latent toxoplasmosis seems to have no significant negative impact on the health of the offspring. However, pregnant women with latent toxoplasmosis have been reported to have seemingly younger (less developed) fetuses, especially at the 16th week of pregnancy (Flegr et al. 2005, Kaňková and Flegr 2007). The immunological hypothesis assumes that $T$. gondii could weaken or switch off the mechanism of spontaneous abortions, which is, under normal conditions, responsible for the elimination of embryos with developmental defects and a (statistically) slower fetal growth rate. The hypothesis was later supported by the observed increase in 
the secondary sex ratio in children of women with latent toxoplasmosis (Kaňková et al. 2007a). The probability of the birth of a boy increased up to 0.71 , which means that about 250 boys were born for every 100 girls to women with moderate concentrations of anti-Toxoplasma antibodies (and probably with recent but already latent infection). This effect of latent toxoplasmosis was later confirmed in experimentally infected mice (Kaňková et al. 2007b). Mice with toxoplasmosis had a higher sex ratio (expressed as the proportion of males in the offspring) than controls in the early phase of latent infection. One of possible physiological mechanisms that could be responsible for $T$. gondii-associated increase of the sex ratio is an elevated concentration of glucose (Cameron 2004, Cameron et al. 2008).

Significantly lower levels of antibodies against $T$. gondii infection were detected in the autoimmune disease type 1 diabetes mellitus (T1DM) patients (Krause et al. 2009). This phenomenon in T1DM patients may be related to their younger ages, but may also point to a protective role of the infections in T1DM development in susceptible individuals. However, it has been shown that there are considerable similarities in the genetic and environmental risk factors between latent $T$. gondii infection and diabetes mellitus (Prandota 2013).

Gestational diabetes mellitus (GDM) is a form of diabetes that is diagnosed during pregnancy and increases the risk of pregnancy and fetal complications (Johns et al. 2006, Vohr and Boney 2008, Vambergue et al. 2008). Immediately after pregnancy, five to 10 percent of women with GDM are diagnosed with diabetes, usually type 2, and women with a history of GDM have $35-60 \%$ chance of developing diabetes in the next 10 to 20 years (Centers for Disease Control and Prevention, 2011). It is possible that both the toxoplasmosis-associated increase of the sex ratio and higher incidence of GDM in high-risk T. gondii-positive subjects may be mediated by an effect of $T$. gondii on the concentration of blood glucose.

The main purpose of the present study is to search for differences in the blood glucose levels between $T$. gondii-negative and T. gondii-positive pregnant women and to test the hypothesis that GDM is more often diagnosed in $T$. gondii-positive subjects.

\section{MATERIALS AND METHODS}

\section{Patients}

The experimental design was a retrospective cross-sectional study. The main data set covered 199 pregnant women screened for various clinical reasons for GDM in the 24-28th gestational weeks in the General University Hospital in Prague in 2008. The oral glucose tolerance test (OGTT) consisted of measurements of blood glucose in three blood samples from each woman collected: A) at baseline after eight hours of fasting, B) 60 min after drinking a measured dose of glucose solution, C) $120 \mathrm{~min}$ after drinking the measured dose of glucose solution. The second data set covered 89 women whose GDM status was known but whose tolerance test data (blood glucose concentrations) were not available. The third data set included information from a questionnaire survey of 1381 women from the hospital databases. For various technical reasons, the questionnaire only reached about 1200 women. The women were asked, among other questions unrelated to the present study, whether they had gestational diabetes mellitus during pregnancy. Altogether 249 respondents not included in data sets 1 and 2 provided either a positive or negative answer.

Serum samples collected from pregnant women at the time of screening for chromosomal abnormalities, routinely performed in the 9-12th gestational weeks in this Hospital, were frozen and stored at $-70^{\circ} \mathrm{C}$ for later analyses. We used these samples for the retrospective detection of antibodies against $T$. gondii.

During the whole time of the study, we worked with an anonymised data set. The study was approved by the Ethical Committee of the General University Hospital and the First Faculty of Medicine of the Charles University in Prague.

\section{Investigations on oral glucose tolerance test}

Laboratory assessment was performed in a single laboratory at the Institute of Clinical Biochemistry and Laboratory Diagnostics, General University Hospital in Prague. The exclusion criterion was an acute illness like flu or other infectious disease. The women were allowed an unrestricted carbohydrate diet for 3 days prior to the oral glucose tolerance test and were requested not to eat at least eight hours before the test. They were instructed not to take their morning medication, but to wait and take it after the test is over. Blood samples were collected by venipuncture. The women were given a $75 \mathrm{~g}$ glucose drink to be consumed in $5 \mathrm{~min}$. The first baseline blood sample was taken prior to the administration of glucose. The second and third blood samples were taken at $60 \mathrm{~min}$ and $120 \mathrm{~min}$ after glucose administration, respectively. The women remained seated and stayed in the department while undergoing the test. If some glucose was lost by vomiting, the test was terminated.

For the Czech population, the normal glucose levels in the blood range up to $5.5 \mathrm{mmol} / \mathrm{l}$ of fasting, $8.8 \mathrm{mmol} / \mathrm{l}$ at $60 \mathrm{~min}$ after the glucose drink and $7.6 \mathrm{mmol} / \mathrm{l}$ at $120 \mathrm{~min}$ after the glucose drink. A positive screening result for gestational diabetes mellitus was defined as an increase of any of these levels of more than $0.1 \mathrm{mmol} / \mathrm{l}$ above the norm (Andělová 2013).

\section{Immunological tests for toxoplasmosis}

The complement-fixation test (CFT) which determines the overall levels of IgM and IgG antibodies of particular specificity, and Enzyme-Linked Immunosorbent Assays (ELISA) (IgG ELISA: SEVAC, Prague, IgM ELISA: TestLine, Brno) were used to detect toxoplasmosis status of the women. Using ELISA, specific classes of antibodies ( $\mathrm{IgG}, \mathrm{IgM}$ ) enabled to discriminate between the acute and latent phases of the infection. Positivity for toxoplasmosis was defined as a CFT titre of $1: 4$ and more, together with an index of positivity (IP) of $>1.2$ for IgG ELISA antibodies against $T$. gondii.

\section{Statistics}

The statistical program Statistica 9.0 was used for all testing. The influence of latent toxoplasmosis on the blood glucose level was first evaluated using the General Linear Model (GLM) repeated measure analysis, with three dependent variables, namely the blood glucose levels after $8 \mathrm{~h}$ of fasting and $60 \mathrm{~min}$ and $120 \mathrm{~min}$ after the glucose drink as repeated measures. Subsequently, these 


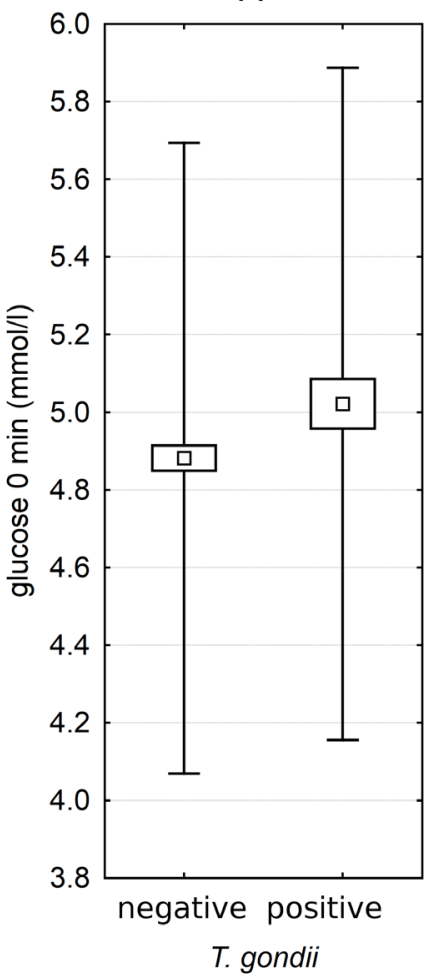

B

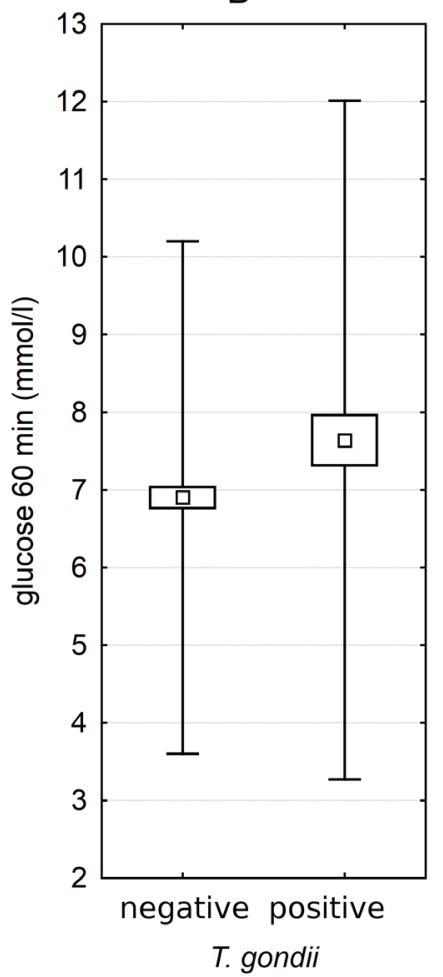

C

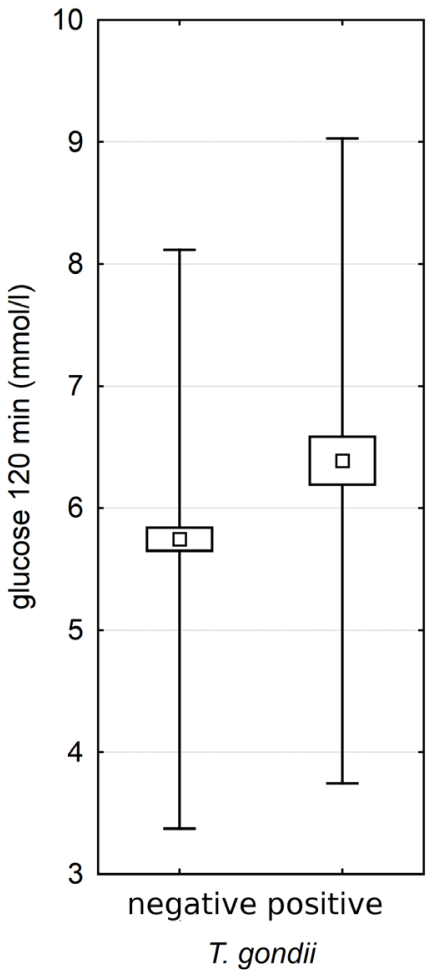

Fig. 1. Differences in the blood glucose levels between $T$. gondii-positive and $T$. gondii-negative pregnant women screened for gestational diabetes (24-28th gestational weeks). The boxes and spreads show standard error and standard deviation ranges, respectively. A - baseline glucose levels after 8 hours of fasting $(0 \mathrm{~min}) ; \mathbf{B}$ - glucose levels 60 min after the glucose drink; $\mathbf{C}-$ glucose levels 120 min after the glucose drink.

three dependent variables were also evaluated separately using a simple GLM technique. An independent continuous variable, maternal body weight in the 9-12th gestational weeks, was included in all models. The influence of the binary variable latent toxoplasmosis on the probability of a positive GDM screening result was evaluated using the contingency table method. Four women with twin pregnancies were excluded from the analysis. For a particular category of analysis, some data were not available and the numbers of women varied between analyses.

\section{RESULTS}

The final data set covered 195 pregnant women. However, all three measurement data were available only for 191 of them. GLM with the blood glucose levels after $8 \mathrm{~h}$ of fasting and $60 \mathrm{~min}$ and $120 \mathrm{~min}$ after the glucose drink as repeated measures and independent binary variables toxoplasmosis and maternal body mass showed that Toxoplasma gondii-positive women had higher glucose levels in the blood $\left(\mathrm{n}=191, \mathrm{p}=0.010\right.$, eta $\left.{ }^{2}=0.024\right)$ than $T$. gondii-negative women. The effect of the continuous predictor maternal body mass was positive and also significant $(\mathrm{p}=0.003$, eta $\left.^{2}=0.030\right)$.

The univariate GLM analysis with the glucose level as the dependent variable and binary variable toxoplasmosis and continuous variable maternal weight in the 9-12th gestational weeks as independent variables showed that 45 T. gondii-positive women had higher levels of fasting plasma glucose (OGTT $0 \mathrm{~min}$ ) (mean = $5.04 \mathrm{mmol} / \mathrm{l}$; $\mathrm{CI}_{95}=4.92$, 5.17) than $149 \mathrm{~T}$. gondii-negative wom- en $\left(\right.$ mean $\left.=4.88 \mathrm{mmol} / \mathrm{l} ; \mathrm{CI}_{95}=4.82,4.95\right)(\mathrm{p}=0.026$, eta $^{2}=0.026$ ) (Fig. 1A). A positive correlation also existed between maternal weight in the 9-12th gestational weeks $\left(\mathrm{p}<0.001\right.$, eta $\left.^{2}=0.145\right)$. The same model was used for evaluating the effect of toxoplasmosis on the blood glucose level at 1 hour (OGTT $60 \mathrm{~min}$ ) or two hours (OGTT 120 min) after the glucose drink (Fig. 1B,C).

Sixty min after the glucose drink, 45 T. gondii-positive mothers had higher blood glucose levels (mean $\left.=7.73 \mathrm{mmol} / 1 ; \mathrm{CI}_{95}=7.09,8.37\right)$ than $147 \mathrm{~T}$. gondii-negative mothers $\left(\right.$ mean $=6.89 \mathrm{mmol} / 1 ; \mathrm{CI}_{95}=6.61$, $7.16),\left(p=0.007\right.$, eta $\left.^{2}=0.038\right)$ and the effect of the continuous predictor maternal weight was again significant $\left(\mathrm{p}=0.002\right.$, eta $\left.^{2}=0.051\right)$.

Two hours (120 min) after the glucose drink, 44 T. gondii-positive mothers had higher blood glucose levels (mean $\left.=6.43 \mathrm{mmol} / \mathrm{l} ; \mathrm{CI}_{95}=6.03,6.83\right)$ than $148 \mathrm{~T}$. gondii-negative mothers $\left(\right.$ mean $=5.74 \mathrm{mmol} / \mathrm{l} ; \mathrm{CI}_{95}=5.55$, $5.93),\left(\mathrm{p}=0.001\right.$, eta $\left.{ }^{2}=0.053\right)$, but the effect of the continuous predictor maternal body mass was not significant $\left(\mathrm{p}=0.22\right.$, eta $\left.^{2}=0.008\right)$.

The GLM analysis showed a positive correlation of anti-T. gondii IgG antibodies and blood glucose level at $120 \mathrm{~min}$ after the glucose drink for $45 T$. gondii-positive women (partial Kendall test with maternal age as a covariate: $p=0.049$; Fig. 2). This correlation was not significant either at $0 \min (p=0.456)$ or $60 \min (p=0.206)$, neither was the correlation between the concentration of IgG antibodies and blood glucose level and the concentration of an- 


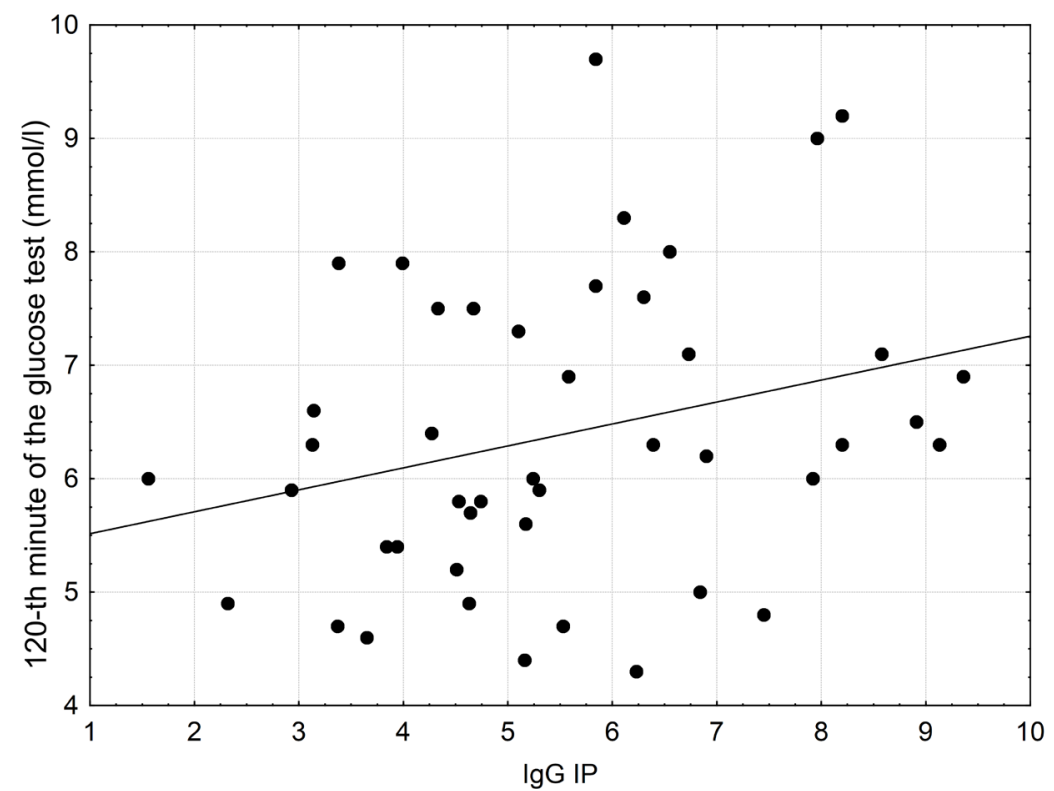

Fig. 2. Correlation of anti-Toxoplasma gondii IgG antibody levels (index of positivity) and blood glucose at 120 minutes after the glucose drink in T. gondii-positive pregnant women screened for gestational diabetes (24-28th gestational weeks).

ti-T. gondii IgG antibodies at any minute after the glucose drink for T. gondii-negative women (partial Kendall test with maternal age as a covariate $0 \mathrm{~min}: \mathrm{n}=151, \mathrm{p}=0.618$; 60 min: $\mathrm{n}=149, \mathrm{p}=0.676 ; 120 \min : \mathrm{n}=150, \mathrm{p}=0.979$ ).

The difference in the prevalence of gestational diabetes mellitus between $T$. gondii-positive and $T$. gondii-negative women was searched for in a combined set of 532 women whose OGTT data were available. Of 409 T. gondii-negative women, 49 (12.0\%) were screened positive for GDM while the GDM prevalence rate in $123 \mathrm{~T}$. gondii-positive women was $19.5 \%$ (24 cases). This difference was significant $\left(\mathrm{n}=532, \chi^{2}=4.53, \mathrm{p}=0.033\right.$, odds ratio $\left.=1.78\right)$.

\section{DISCUSSION}

Pregnant women with latent toxoplasmosis had significantly higher blood glucose levels in the oral glucose tolerance test at baseline after 8 hours of fasting and at 60 and 120 minutes after the 75 g glucose drink. The glucose level positively covaried with the concentration of anti-Toxoplasma gondii antibodies, suggesting that the effects of toxoplasmosis decreased with the time from infection. Toxoplasma gondii-infected women showed higher prevalence of gestational diabetes than $T$. gondii-free women.

Our results, namely the increased blood glucose level and higher incidence of GDM in $T$. gondii-infected women could also have considerable clinical impact. For example, these associations may provide new clues to the understanding of the complex pathogenesis of GDM. This disorder has important short-term as well as long-term consequences for women and their offspring. The major of them is a predisposition to the development of metabolic syndrome and type 2 diabetes. Even in developed countries, women with GDM may remain undiagnosed despite the fact that a proper lifestyle intervention has been documented to prevent or at least reduce the development of these severe conditions. It would be therefore useful to examine
T. gondii-positive mothers for GDM during the first trimester. This is probably especially true in the countries with high prevalence of toxoplasmosis or in countries where the screening for GDM in pregnancy is mandatory (e.g in the Czech Republic).

The extensive genetic heterogeneity of $T$. gondii strains also needs to be taken into account. Recent studies have shown that the strains of this parasite may differ dramatically in their impact on the physiology, health and also behaviour of the infected host (Morisset et al. 2008, Kannan 2010, McLeod 2012). In the future studies, it would be desirable to perform serological or even genetic typing of T. gondii isolates. Two-tailed statistical tests demonstrated a significant correlation between the concentrations of specific antibodies and glucose 120 minutes, but not at the 60 minutes after the glucose drink and at baseline (0 minute) before the glucose drink. It suggests that this effect might be probably linked to an increase in insulin resistance.

The present study also showed higher prevalence of GDM in T. gondii-infected women. Toxoplasma gondii-positive women had higher body mass before pregnancy than T. gondii-negative women (Flegr et al. 2005, Kaňková et al. 2010). It is known that high maternal weight is associated with a substantially higher risk of GDM (Chu et al. 2007). The effect of T. gondii positivity on higher prevalence of GDM could just be a side-effect of the influence of toxoplasmosis on body weight. Our data, however, did not support this hypothesis as the effect of latent toxoplasmosis on the higher blood glucose level was significant in the model with body mass as a covariate.

The original aim of the present study was to find the mechanisms underlying the observed associations of the sex ratio and GDM with $T$. gondii infection (Cameron 2004, Cameron et al. 2008). Latent toxoplasmosis increases the secondary sex ratio in humans and laboratory mice 
(Kaňková et al. 2007a,b). The physiological mechanism of this effect is unknown; however, we hypothesise that a manipulation of the blood glucose concentration could play a role in it (Larson et al. 2001).

Research on the role of glucose in the reproductive functions has shown that excess glucose favours the development of male blastocysts, providing a potential mechanism for sex-ratio variation in relation to maternal condition around conception (Cameron 2004). Also, the change in glucose levels resulted in a change in sex ratios, with more daughters being born to females with experimentally lowered glucose (Cameron et al. 2008). A study using mice as an animal model for human diabetes has found that an increase in circulating glucose resulted in a male-biased sex ratio (Machado et al. 2001).

Our present study suggests that $T$. gondii-infected women have increased blood glucose levels, which is in accordance with our hypothesis suggesting that the glucose concentration plays a role in $T$. gondii-associated sex ratio shifts. This hypothesis is also supported by the existence of a positive correlation between the concentration of anti-T. gondii IgG antibodies and the blood glucose concentration. The concentration of anti-T. gondii IgG antibodies is known to decrease with the time passed since infection (Kodym et al. 2007). In the early phase of latent toxoplasmosis, i.e. in mice that delivered in the fourth month of infection, the sex ratio was higher than in controls, whereas the mice in a later phase of infection, i.e. those that delivered in the fifth to eighth month of infection, had a lower sex ratio than controls (Kaňková et al. 2007b).

In a large cross-sectional study in a sample of $1803 \mathrm{cli}-$ ents, the probability of the birth of a boy was also increased in women with the highest concentrations of anti- $T$. gondii antibodies while decreased in those with a low concentration of anti-Toxoplasma antibodies (Kan̆ková et al. 2007a). The reduction of the positive effect of $T$. gondii on the concentration of glucose with decrease of anti-T. gondii $\operatorname{IgG}$ antibody level, which was observed in the present study, supports the hypothesis that the glucose could be the proximate cause of the toxoplasmosis-associated changes in the offspring sex ratio.
Recently, it was suggested that maternal $\mathrm{T}$ and potentially also B cells transferred during pregnancy and/or the breast milk feeding and their encounter with the antigen in mesenteric lymph nodes might play a role in development of T1DM (Roy et al. 2011). Maternal microchimerism in peripheral blood was demonstrated to be significantly higher in patients with T1DM compared to unaffected siblings and healthy subjects (Nelson et al. 2007). It was suggested that Toxoplasma-containing cells of fetal origin could disseminate the parasite in the maternal organism (Prandota 2012). It has been reported recently that Toxoplasma increases migration activity of infected leukocytes, which could help spread infection in various tissues of the host's body (Fuks et al. 2012).

Toxoplasma gondii infection during gestation and/or after birth may be responsible for development of both T1DM and type 2 diabetes mellitus (T2DM) in children, adolescents and adults (Prandota 2013). Moreover, a significantly lower occurrence of antibodies against $T$. gondii found in the sera of patients with T1DM compared with their first-degree family members or healthy controls may be due to their $\mathrm{T}$ and/or $\mathrm{B}$ cell exhaustion perspective caused by chronic infection with the parasite (Krause et al. 2009). In contrast, patients with T2DM had increased anti- $T$. gondii antibodies significantly more frequently than respective controls (Gokce et al. 2008). Impaired vascular endothelial function characteristic for the patients with diabetes mellitus may be at least in part due to the preferential T. gondii infection of endothelial cells (Prandota 2013).

However, it must be reminded that all previous studies have focused on the effect of the glucose concentration on the sex ratio at the time of conception, while we addressed the concentration of glucose during pregnancy. Further work would be necessary to confirm our results in a population of non-pregnant women in child-bearing age.

Acknowledgements. The research was supported by research grants UNCE 204004 and PRVOUK 25 from the Charles University, Prague and RVO-VFN64165 from the Ministry of Health of the Czech Republic.

\section{REFERENCES}

ANDĚLOVÁ K. 2013: [Administration of an impaired glucose tolerance test (screening) in pregnancy - the recommended practice]. Čes. Gynekol. 78: 10. (In Czech.)

Baettie C.P. 1982: The ecology of toxoplasmosis. Ecol. Dis. 1: 13-20.

CAmeron E.Z. 2004: Facultative adjustment of mammalian sex ratin in support of the Travers-Willard hypothesis: evidence for a mechanism. Proc. R. Soc. B 271: 1723-1728.

Cameron E.Z., Lemons P.R., Bateman P.W, Bennett N.C. 2008: Experimental alteration of liter sex ratios in a mammal. Proc. R. Soc. B 275: 323-327.

Centers for Disease Control and Prevention 2011: National diabetes fact sheet: general information and national estimates on diabetes in the United States. Atlanta, GA: U.S. Department of Health and Human Services, Centers for Disease Control and Prevention, $6 \mathrm{pp}$
Chu S.Y., Callaghan W.M., Kim S.Y., Schmidt C.H., Lau J., England J.L., Dietz P.M. 2007: Maternal obesity and risk of gestational diabetes mellitus. Diabetes Care 30: 2070-2076.

FLEGR J. 2013: Influence of latent Toxoplasma infection on the human personality, physiology, and morphology: pros and cons of the Toxoplasma-human model in studying the manipulation hypothesis. J. Exp. Biol. 216: 127-133.

Flegr J., Hrdé Š., Kodym P. 2005: Influence of latent toxoplasmosis on human health. Folia Parasitol. 52: 199-204.

Flegr J., Prandota J., SovičKová M., Israili Z.H. 2014: Toxoplasmosis - a global threat. Correlation between latent toxoplasmosis and specific disease burden - a WHO data-based study of 29 European and 59 non-European countries. PLoS ONE 9: e90203.

Flegr J., Zítková S., Kodym P., Frynta D. 1996: Induction of changes in human behaviour by the parasitic protozoan Toxoplasma gondii. Parasitology 133: 49-54. 
Fuks J.M., Arrighi R.B., Weidner J.M., Kumar Mendu S., Jin Z., Wallin R.P.A., Rethi B., Birnir B., Barragan A. 2012: GABAergic signaling is linked to a hypermigratory phenotype in dendritic cells infected by Toxoplasma gondii. PLoS Pathog. 8: e1003051.

Gokce C., Yazar S., Bayram F., Gundogan K., Yaman O., SAHIN I. 2008: Anti-Toxoplasma gondii antibodies in type 2 diabetes. Natl. Med. J. India 21: 51.

Johns K., Olynik C., Mase R., Kreisman S., Tildesley H. 2006: Gestational diabetes mellitus outcome in 394 patients. J. Obstet. Gynaecol. Can. 28: 122-127.

Jones J.L., Kruszon-Morgan D., Wilson M, McQuillan G., Navis T., McAuley J.B. 2001: Toxoplasma gondii infection in the United States: seroprevalence and risk factors. Am. J. Epid. 4: $357-365$.

KaŇKovÁ Š., Flegr J. 2007: Longer pregnancy and slower fetal development in women with latent "asymptomatic" toxoplasmosis. BMC Infect. Dis. 7: 114

Kaňková Š., Kodym P., Frynta D., Vavřinová R., Kuběna A., FLEGR J. 2007b: Influence of latent toxoplasmosis on the secondary sex ratio in mice. Parasitology 134: 1709-1717.

KAŇKOVÁ Š., ŠULC J., FLEGR J. 2010: Increased pregnancy weight gain in women with latent toxoplasmosis and RhD-positivity protection against this effect. Parasitology 137: 1773-1779.

Kaňková Š., Šulc J., Nouzová K., Fajfrlík K., Frynta D., FLEGR J. 2007a: Women infected with parasite Toxoplasma have more sons. Naturwissenschaften 94: 122-127.

Kannan G., Moldovan K., Xiao J.C., Yolken R.H., Jones-Brando L., Pletnikov M.V. 2010: Toxoplasma gondii strain-dependent effects on mouse behaviour. Folia Parasitol. 57: 151-155.

Kodym P., Machala L., Roháčová H., Širocká B., Malý M. 2007: Evaluation of a commercial IgE ELISA in comparison with IgA and IgM ELISAs, IgG avidity assay and complement fixation for the diagnosis of acute toxoplasmosis. Clin. Microbiol. Infec. 13: 40-47.

Krause I., Anaya J.M., Fraser A., Barzilai O., Ram M., Abad V., Arango A., García J., Shoenfeld Y. 2009: Anti-infectious antibodies and autoimmune-associated autoantibodies in patients with type I diabetes mellitus and their close family members. Ann. N. Y. Acad. Sci. 1173: 633-639.

Larson M.A., Kimura K., Kubisch H.M., Roberts R.M. 2001: Sexual dimorphism among bovine embryos in their ability to make the transition to expanded blastocyst and in the expression of the signaling molecule IFN-tau. Proc. Natl. Acad. Sci. USA 98: 9677-9682.

Lindová J., Novotná M., Havlíček J., Jozífková E., Skallová A., Kolbeková P., Hodný Z., Kodym P., Flegr J. 2006: Gender differences in behavioural changes induced by latent toxoplasmosis. Int. J. Parasitol. 36: 1485-1492.

Machado A.F., Zimmerman E.F., Hovland D.N., Weiss R, Collins M.D. 2001: Diabetic embryopathy in C57BL/6J mice: altered fetal sex ratio and impact of the Splotch gene. Diabetes 50: $1193-1200$.

McLeod R., Boyer K.M., Lee D., Mui E., Wroblewski K., Harrison T., Noble A.G., Withers S., Swisher Ch.N., Heydemann P.T., Sautter M., Babiarz J., Fabian P., Meier P., Grigg M.E., the Toxoplasmosis Study Group 2012: Prematurity and severity are associated with Toxoplasma gondii alleles (NCCCTS, 1981-2009). Clin. Infect. Dis. 54: 1595-1605.

Morisset S., Peyron F., Lobry J.R., Garweg J., Ferrandiz J., Musset K., Gomez-Marin J.E., de la Torre A., Demar M., Carme B., Mercier C., Garin J.F., Cesbron-Delauw M.F. 2008: Serotyping of Toxoplasma gondii: striking homogeneous pattern between symptomatic and asymptomatic infections within Europe and South America. Microbes Infect. 10: 742-747.

Nelson J.L., Gillespie K.M., Lambert N.C., Stevens A.M., Loubiere L.S., Rutledge J.C., Leisenring W.M., Erickson T.D., Yan Z., Mullarkey M.E., Gale E.A.M. 2007: Maternal microchimerism in peripheral blood in type 1 diabetes and pancreatic islet beta cell microchimerism. Proc. Natl. Acad. Sci. USA 104: 1637-1642.

Prandota J. 2012: Increased generation of antibodies and autoantibodies directed against brain proteins in patients with autism and their families may be caused by T. gondii infection. Maternal and fetal microchimerisms probably play an important role in these processes acting as a "Trojan horse" in dissemination of the parasite. In: C. Gemma (Ed.), Neuroinflammation Pathogenesis, Mechanisms and Management. Nova Science Publishers, New York, pp. 447-638.

Prandota J. 2013: T. gondii infection acquired during pregnancy and/or after birth may be responsible for development of both type 1 and 2 diabetes mellitus. J. Diabet. Metab. 4: 55.

Roy E., Leduc M., Guegan S., Rachdi L., Kluger N., Scharfmann R., Aractingi S., Khosrotehrani K. 2011: Specific maternal microchimeric T cells targeting fetal antigens in $\hat{I}^{2}$ cells predispose to autoimmune diabetes in the child. J. Autoimmun. 36: $253-262$.

Tenter A.M., Heckeroth A.R., Weiss L.M. 2000: Toxoplasma gondii: from animals to humans. Int. J. Parasitol. 30: 1217-1258.

Vambergue A., Dognin C., Boulogne A., Re'jou M.C., BiauSQUE S., FONTAINE P. 2008: Increasing incidence of abnormal glukose tolerance in women with prior abnormal glucose tolerance during pregnancy: DIAGEST 2 study. Diabet. Med. 25: $58-64$.

Vohr B.R., Boney C.M. 2008: Gestational diabetes: the forerunner for the development of maternal and childhood obesity and metabolic syndrome? J. Matern. Fetal. Neonatal. Med. 21: 149-157.

Wolf A., Cowen D., Paige B.H. 1939: Toxoplasmic encephalomyelititis. III. A new case of granulomatoces encephalomyelititis due to a protozoon. Am. J. Pathol. 15: 657.

Cite this article as: Kaňková Š., Flegr J., Calda P. 2015: An elevated blood glucose level and increased incidence of gestational diabetes mellitus in pregnant women with latent toxoplasmosis. Folia Parasitol. 62: 056. 